\title{
Existence of minimizers for a polyconvex energy in a crystal with dislocations
}

\author{
Stefan Müller • Mariapia Palombaro
}

Received: 25 February 2007 / Accepted: 18 May 2007 / Published online: 13 July 2007

C) Springer-Verlag 2007

\begin{abstract}
We provide existence theorems in nonlinear elasticity for minimum problems modeling the deformations of a crystal with a given dislocation. A key technical difficulty is that due to the presence of a the dislocation the elastic deformation gradient cannot be in $L^{2}$. Thus one needs to consider elastic energies with slow growth, to which the original results of Ball cannot be applied directly.
\end{abstract}

Mathematics Subject Classification (2000) 74 N05 - 58A25 - 74B20 - 49J45 - 46E40

\section{Introduction}

The purpose of this article is to present existence theorems for problems associated with the minimization of the elastic energy stored in a crystal in presence of dislocations. The setting is that of nonlinear elasticity.

Dislocations represent an important class of defects in crystalline solids and are very often the primary agents of plasticity. See for example $[8,11]$ for a general survey on this subject. The constituent atoms of a crystal are arranged in a pattern that repeats itself periodically in the space forming a three-dimensional lattice. Dislocations occur when atomic planes slide over each other on so-called slip planes. The extent to which one part of the crystal has slipped relative to the other is determined by a translation vector $\mathbf{b}$, called the Burgers vector.

Here we take a continuum point of view which goes back to Volterra. In this setting the dislocation may be thought of as the result of cutting the crystal across a surface $\mathcal{S}$ in the slip plane, imposing a relative translation b between the upper and the lower part of $\mathcal{S}$ and rejoining the cut region. This leads to the definition of a dislocation loop as the boundary

S. Müller $(\bowtie) \cdot$ M. Palombaro

Max Planck Institute for Mathematics in the Sciences, Inselstr. 22-26,

04103 Leipzig, Germany

e-mail: sm@mis.mpg.de

M. Palombaro

e-mail: mariapia.palombaro@mis.mpg.de 
that separates the region $\mathcal{S}$ on the slip plane which has undergone slip from the region that has not (the curve $\Gamma$ in Fig. 1a).

More precisely, we consider a bounded region $\Omega \subset \mathbb{R}^{3}$ which represents the reference configuration of the crystal and we look at deformations $u: \Omega \rightarrow \mathbb{R}^{3}$. In a modern view of the Volterra picture [10] $u$ is a special map of bounded variations (SBV, see, e.g., [1]) and its distributional deformation gradient $D u$ consists of a singular part due to the jump across $\mathcal{S}$ and an absolutely continuous part (with respect to Lebesgue measure)

$$
D u=\nabla u d x+\mathbf{b} \otimes v d \mathcal{H}^{2}\llcorner\mathcal{S} .
$$

Following [10] we interpret $\nabla u$ as the elastic deformation. As usual we assume that the elastic energy has the form

$$
\int_{\Omega} W(\nabla u) d x,
$$

where the function $W: \mathbb{M}^{3 \times 3} \rightarrow \mathbb{R} \cup\{+\infty\}$ is polyconvex and is allowed to take the value $+\infty$ to incorporate constraints like det $D u>0$, which prevents interpenetration of matter.

Taking the curl of Eq. (1.1) in the sense of distributions yields the relation

$$
\operatorname{curl}(\nabla u d x)=-\mathbf{b} \otimes \dot{\Gamma} d \mathcal{H}^{1}\llcorner\Gamma .
$$

The above formula suggests to minimize (1.2) over those fields $F: \Omega \rightarrow \mathbb{M}^{3 \times 3}$ whose curl is concentrated along the curve $\Gamma$ :

$$
\operatorname{curl} F=-\mathbf{b} \otimes \dot{\Gamma} d \mathcal{H}^{1}\llcorner\Gamma
$$

in the sense of distributions. Note that the surface $\mathcal{S}$ no longer appears explictly in this formulation. Indeed, for any surface $\mathcal{S}^{\prime}$ which has $\Gamma$ as its boundary (with the appropriate orientation) the field $F^{\prime}:=F d x+\mathbf{b} \otimes v^{\prime} d \mathcal{H}^{2}\left\llcorner\mathcal{S}^{\prime}\right.$ is curl-free (in the sense of distributions) and thus the distributional gradient of a deformation $u^{\prime}$, with a jump discontinuity across $\mathcal{S}^{\prime}$. In that sense $F$ and $\Gamma$ are the natural variables, while the choice of a specific cut surface $\mathcal{S}$ is a matter of convenience.

The constraint (1.3) can be expressed by the equivalent condition

$$
\int_{\alpha} F \frac{\dot{\alpha}}{|\dot{\alpha}|}=\mathbf{b} \operatorname{Link}(\alpha, \Gamma)
$$

for every smooth closed curve $\alpha$ contained in $\Omega \backslash \Gamma$ (see Fig. 1b). Here $\operatorname{Link}(\alpha, \Gamma)$ denotes the winding number of the curve $\alpha$ with respect to $\Gamma$.

By (1.4) we also deduce that the admissible fields, i.e., the deformation gradients compatible with the dislocation, cannot expect to be in $L^{p}(\Omega)$ for $p \geq 2$. Indeed if $\alpha$ is a circle of radius $r$ around $\Gamma$, then (1.4) implies that

$$
f_{\alpha} F \frac{\dot{\alpha}}{|\dot{\alpha}|}=\frac{1}{2 \pi r} \mathbf{b} \operatorname{Link}(\alpha, \Gamma) \quad \forall r>0,
$$

which yields

$$
|F(x)| \sim \frac{1}{\operatorname{dist}(x, \Gamma)} .
$$

From (1.5) it is readily seen that the $L^{2}$ norm of $F$ in a cylindrical neighborhood of $\Gamma$ diverges logarithmically. This is exactly why in a linear elastic setting the energy of a dislocation line is 

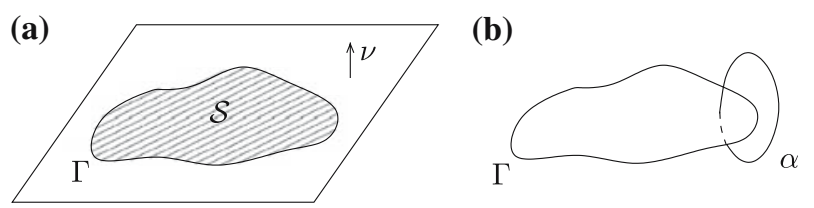

Fig. 1 a The dislocation loop $\Gamma$. b A generic curve $\alpha$ winding around $\Gamma$

infinite. We will avoid this difficulty by considering energy densities $W$ which grow slower than quadratic at $\infty$. We will later use the following growth condition from below (for a suitable $p<2$ )

$$
\forall A \in \mathbb{M}^{3 \times 3} \quad W(A) \geq c\left(|A|^{p}+|\operatorname{adj} A|^{p}+|\operatorname{det} A|^{p}\right)-C .
$$

Here $\operatorname{adj} A$ denotes the matrix of $2 \times 2$ minors.

Summarizing, we address the problem of minimizing

$$
\mathcal{W}(F):=\int_{\Omega} W(F) d x,
$$

over all matrix fields $F \in L^{p}\left(\Omega, \mathbb{M}^{3 \times 3}\right)$ which satisfy the constraint (1.3) and for which $\operatorname{adj} F$ and det $F$ are in $L^{p}$ (further conditions on the precise class of admissible functions will be discussed below). We do not require that $\Gamma$ lies in a plane and allow any curve which is the image of a standard planar $S^{1}$ under a smooth diffeomorphism $\mathbb{R}^{3} \rightarrow \mathbb{R}^{3}$.

The fact that the exponent $p$ in the growth condition is restricted to $p<2$ does not allow us to use Ball's original existence results [2], which require $W(A) \geq c|A|^{q}-C$, with $q \geq 3$. For $2 \leq p \leq 3$ extensions due to Šverák [12] and Conti and De Lellis [3] are available, but these do not apply for $p<2$. We will still follow Ball's strategy of proof using the refined settings of [9] and [6], respectively. More precisely, we establish the existence of minimizers for (1.7) in two different classes. In the first existence result, Theorem 3.4, that holds for $\frac{3}{2}<p<2$, we require the admissible fields be locally the gradients of Sobolev functions for which the distributional Jacobian determinant and the distributional Jacobian cofactors are represented by functions.

In the second existence result, Theorem 4.6, we work in a class of fields which are locally the gradients of Cartesian maps. In this context one needs to use the notion of rectifiable currents and related compactness results due to Giaquinta et al. [6]. In this setting we consider the range $1<p<2$.

\section{Assumptions and notations}

We assume that the function $W: \mathbb{M}^{3 \times 3} \rightarrow \mathbb{R} \cup\{+\infty\}$ is polyconvex, i.e., there exists a convex function $g: \mathbb{R}^{19} \rightarrow \mathbb{R} \cup\{+\infty\}$ such that

$$
W(A)=g(A, \operatorname{adj} A, \operatorname{det} A) \quad \forall A \in \mathbb{M}^{3 \times 3} .
$$

We assume that $g$ is lower semicontinuous and $W$ satisfies the following growth condition

$$
W(A) \geq c\left(|A|^{p}+|\operatorname{adj} A|^{p}+|\operatorname{det} A|^{p}\right)-C \quad \forall A \in \mathbb{M}^{3 \times 3},
$$

for some positive constants $c$ and $C$. 
We denote by $\Omega$ an open bounded set of $\mathbb{R}^{3}$. The curve $\Gamma \subset \Omega$ is assumed to be smooth, closed and diffeomorphic to the standard $S^{1}$. More precisely we assume that there exists a diffeomorphism $\psi: \mathbb{R}^{3} \rightarrow \mathbb{R}^{3}$ such that $\psi(\Gamma)=S^{1}$. In particular $\Gamma$ is unknot.

For a matrix $A, \operatorname{adj} A$ denotes the transpose of its cofactors, so that $A \operatorname{adj} A=(\operatorname{det} A) I$. We will use the minors of a matrix and therefore we introduce the following notation. Let $I, J$ be ordered multiindices, $I=\left\{i_{1}, \ldots, i_{r}\right\}, 1 \leq i_{1}<\cdots<i_{r} \leq 3$. We write $|I|=r$ and we denote by $\bar{I}$ the index which complements $I$ in $\{1,2,3\}$ in the natural increasing order. For $|I|=|J|=r$, let $A_{I J}$ be the submatrix of $A$ consisting of rows $i_{1}, \ldots, i_{r}$ and columns $j_{1}, \ldots, j_{r}$. We set $M_{I}^{J}(A)=\operatorname{det} A_{I}^{J}$. We denote by $A^{i}$ the $i$ th column of the matrix $A$.

Differentiation with respect to the variable $x_{i}$ is denoted by ${ }_{, i}$. Finally $\left\{\rho_{\delta}\right\}_{\delta>0}$ is a sequence of standard smooth convolution kernels in $\mathbb{R}^{3}$, i.e., $\rho_{\delta}(x)=\delta^{-3} \rho(x / \delta), \rho \geq 0, \int \rho=1$, Spt $\rho \subset\{|x|<1\}$.

\section{Main result}

In the present section we provide an existence theorem for the minimizers of (1.7) over a class of fields which are locally the gradients of Sobolev functions satisfying certain differential properties and which are subject to the constraint (1.3) that keeps track of the presence of a dislocation. In order to define the set of such fields, we need to recall the notion of distributional determinant.

Let $u \in W^{1, p}\left(\Omega, \mathbb{R}^{3}\right), p \geq 1$, and assume that $u^{1}(\operatorname{adj} D u)^{1} \in L^{1}\left(\Omega, \mathbb{R}^{3}\right)$. Then the distributional determinant of $D u$, denoted by Det $D u$, is defined as the distribution

$$
\text { Det } D u=\operatorname{div}\left(u^{1}(\operatorname{adj} D u)^{1}\right) \text {, }
$$

i.e.,

$$
\langle\operatorname{Det} D u, \varphi\rangle:=-\int_{\Omega} u^{1}(\operatorname{adj} D u)^{1} \cdot D \varphi d x, \quad \forall \varphi \in \mathcal{D}\left(\Omega, \mathbb{R}^{3}\right) .
$$

In general Det $D u$ is not a function and Det $D u \neq \operatorname{det} D u$.

Similarly, we denote by Adj $D u$ the distribution defined by expressing each of the subdeterminants of $D u$ as the divergence of an $\mathbb{R}^{2}$-valued map. For instance

$$
(\text { Adj } D u)_{11}:=\left(u^{2} u_{, 3}^{3}\right)_{, 2}-\left(u^{2} u_{, 2}^{3}\right)_{, 3} \text {. }
$$

We are now ready to introduce the set $\mathcal{F}$ of admissible functions:

$$
\mathcal{F}:=\left\{F \in L^{p}\left(\Omega, \mathbb{M}^{3 \times 3}\right):|\operatorname{adj} F|, \operatorname{det} F \in L^{p}(\Omega),(\mathbf{i}) \text { and (ii) hold }\right\},
$$

where

(i) $\operatorname{curl} F=-\mathbf{b} \otimes \dot{\Gamma} d \mathcal{H}^{1}\left\llcorner\Gamma\right.$ in $\mathcal{D}^{\prime}(\Omega)$;

(ii) if $\mathcal{B}$ is a ball contained in $\Omega \backslash \Gamma$, then by (i) there exists $u \in W^{1, p}\left(\mathcal{B}, \mathbb{R}^{3}\right)$ (unique up to an additive constant) such that $D u=F$ in $\mathcal{B}$. We require that for all such balls

$$
u \in \mathcal{E}(\mathcal{B}):=\left\{u \in W^{1, p}(\mathcal{B}): \operatorname{Det} D u \in L^{1}(\mathcal{B}), \operatorname{Adj} D u \in L^{1}\left(\mathcal{B}, \mathbb{M}^{3 \times 3}\right)\right\} .
$$

Remark 3.1 Observe that (i) can be replaced by one of the following equivalent conditions:

(ia) for every smooth closed curve $\alpha:[0,1] \rightarrow \Omega \backslash \Gamma$

$$
\int_{0}^{1} F_{\delta}(\alpha(t)) \dot{\alpha}(t) d t \rightarrow \mathbf{b} \operatorname{Link}(\alpha, \Gamma) \text { as } \delta \rightarrow 0,
$$


with $F_{\delta}=F * \rho_{\delta}, \delta<\operatorname{dist}(\alpha, \Gamma)$;

(ib) $\int_{0}^{1} \tilde{F}(\alpha(t)) \dot{\alpha}(t) d t=\mathbf{b} \operatorname{Link}(\alpha, \Gamma)$, for every $\alpha$ such that $\mathcal{H}^{1}$ - a.e. $x \in \alpha$ is a Lebesgue point for $F$ (denoting by $\tilde{F}$ the Lebesgue representative of $F$ ).

Lemma 3.2 Let $\frac{3}{2}<p<2$. Then the set $\mathcal{F}$ is not empty.

Proof We make an explicit construction. Let $D_{1}(O)$ be the disk of radius 1 and center $O=(0,0,0)$ contained in the plane $\left\{x_{3}\right\}=0$, so that $\partial D_{1}(O)=S^{1}$. We first define a map $u \in C_{0}^{\infty}\left(\psi(\Omega) \backslash D_{1}(O), \mathbb{R}^{3}\right)$ and then compose it with $\psi$.

Let $\mathcal{C}_{1}$ be the cone of base $D_{1}(O)$ and vertex $V_{1}=(0,0,1)$ and $\mathcal{C}_{2}$ be the cone of base $D_{1}(O)$ and vertex $V_{2}=(0,0,2)$ as in Fig. 2 . Without loss of generality we may assume that $\mathcal{C}_{1}, \mathcal{C}_{2} \subset \psi(\Omega)$.

Then let $P_{1}=(1,0,0)$ and $P_{2}=\left(\frac{1}{2}, 0,1\right)$. Set $u(x)=0$ in $\psi(\Omega) \backslash \mathcal{C}_{2}$ and $u(x)=\mathbf{b}$ in $\mathcal{C}_{1}$. In $\mathcal{C}_{2}$ we first define $u$ in the triangle $V_{2} O P_{1}$ and then extend it by axial symmetry with respect to the axis $V_{2} O$. In the triangle $V_{1} O P_{1} u$ is already defined and is equal to $\mathbf{b}$. In the triangle $V_{2} V_{1} P_{2}$ let $u$ be the affine interpolation and then in $V_{1} P_{1} P_{2}$ let $u$ be affine in the $x_{1}$ direction. It can be easily checked that $u \in W^{1, p}\left(\psi(\Omega) \backslash D_{1}(O)\right)$ for $p<2$, and that $\operatorname{det} D u=0, \operatorname{adj} D u=0$ a.e. in $\psi(\Omega)$. We can then smoothen $u$ on the lateral surface of $\mathcal{C}_{1}$ and $\mathcal{C}_{2}$ in order to obtain a smooth map in $\psi(\Omega) \backslash D_{1}(O)$ with constant jump equal to $\mathbf{b}$ on $D_{1}(O)$.

In order to obtain a map defined in $\Omega$ and with nonzero Jacobian determinant, we compose $u$ with $\psi$ and add, for instance, the identity map:

$$
\tilde{u}(x):=u(\psi(x))+x .
$$

Next we set $F:=\nabla \tilde{u}$, i.e., $F$ is defined as the absolutely continuous part of the gradient $D \tilde{u}$. The field $F$ constructed in this way satisfies all the desired properties, that is $F \in \mathcal{F}$. Indeed (i) is readily seen to be fulfilled. Moreover if $\mathcal{B} \subset \Omega$ is a ball such that $\psi(\mathcal{B}) \cap D_{1}(O) \neq \emptyset$, then one can define a new potential $\tilde{v} \in \mathcal{E}(\mathcal{B})$ in the following way. Choose a smooth surface $\mathcal{S} \subset \psi(\Omega)$ which has $S^{1}$ as its boundary, i.e., $\partial \mathcal{S}=S^{1}$, and such that $\mathcal{S} \cap \psi(\mathcal{B})=\emptyset$. Suppose that $\mathcal{S}$ lies below $D_{1}(O)$. Then define $v=u+\mathbf{b}$ in the region enclosed by the surfaces $\mathcal{S}$ and $D_{1}(O)$, and $v=u$ elsewhere in $\psi(\Omega)$. We have thus moved the jump of $u$ from $D_{1}(O)$ to $\mathcal{S}$ and the function $v$ defined in this way is smooth in $\psi(\Omega) \backslash \mathcal{S}$. Finally set $\tilde{v}(x):=v(\psi(x))+x$.

The next result provides sufficient conditions under which the distributional determinant is in fact a function. It will be the main tool in the proof of the existence Theorem 3.4.

Fig. 2 The cones $\mathcal{C}_{1}$ and $\mathcal{C}_{2}$

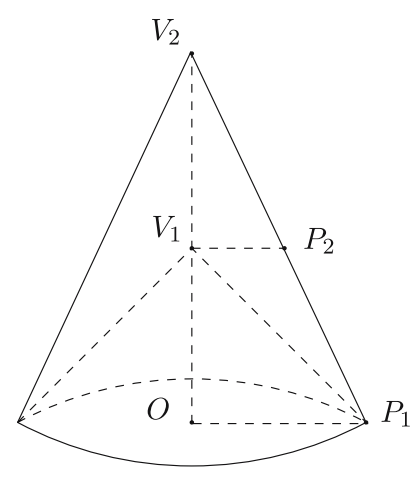


Theorem 3.3 ([9, Theorem 1]) Let $V \subset \mathbb{R}^{3}$ be open, let $1 \leq p<3$ and let $u \in W^{1, p}\left(V ; \mathbb{R}^{3}\right)$.

(i) If $p \geq 3 / 2$ and $\operatorname{Adj} D u \in L^{1}$, then $\operatorname{Adj} D u=\operatorname{adj} D u$.

(ii) If $p \geq 3 / 2$, adj $D u \in L^{p}$ and $\operatorname{Det} D u \in L^{1}$, then $\operatorname{Det} D u=\operatorname{det} D u$.

We are now in the position to state the main result of this section.

Theorem 3.4 Let $\frac{3}{2}<p<2$. Assume that there exists $F \in \mathcal{F}$ such that $\mathcal{W}(F)$ is finite. Then the functional $\mathcal{W}$ attains its minimum on $\mathcal{F}$.

Proof We apply the direct method. In order to exploit the semicontinuity property of the functional (1.7) we will use the gradient structure of the fields $F$ in simply connected sets. Let $\left\{F_{k}\right\}$ be a minimizing sequence. By (2.1) it follows that

$$
\int_{\Omega}\left|F_{k}\right|^{p}+\left|\operatorname{adj} F_{k}\right|^{p}+\left|\operatorname{det} F_{k}\right|^{p}<C
$$

and therefore, up to subsequences, we find

$$
\begin{aligned}
& F_{k} \rightarrow F \text { in } L^{p}\left(\Omega, \mathbb{M}^{3 \times 3}\right), \\
& \operatorname{adj} F_{k} \rightarrow A \text { in } L^{p}\left(\Omega, \mathbb{M}^{3 \times 3}\right), \\
& \operatorname{det} F_{k} \rightarrow D \text { in } L^{p}(\Omega) .
\end{aligned}
$$

If

$$
F \in \mathcal{F}, \quad A=\operatorname{adj} F, \quad D=\operatorname{det} F,
$$

then standard lower-semicontinuity results for convex integrands will imply that

$$
\mathcal{W}(F) \leq \liminf _{k \rightarrow \infty} \mathcal{W}\left(F_{k}\right)=\inf \{\mathcal{W}(F), F \in \mathcal{F}\}
$$

and therefore $F$ will be a minimizer for $\mathcal{W}$.

In order to prove (3.2) it is enough to show that $F \in \mathcal{F}$ and

$$
\left(\operatorname{adj} F_{k}, \operatorname{det} F_{k}\right) \rightarrow(\operatorname{adj} F, \operatorname{det} F) \text { in } \mathcal{D}^{\prime}(\mathcal{B}), \quad \text { for every ball } \mathcal{B} \subset \Omega \backslash \Gamma .
$$

Let $\varphi$ be a smooth function supported in a ball $\mathcal{B} \subset \Omega \backslash \Gamma$. By (ii) it follows that $F_{k}=D u_{k}$ in $\mathcal{B}$ with $u_{k} \in \mathcal{E}(\mathcal{B})$. Since $u_{k}$ is defined only up to an additive constant, we may assume that $u_{k}$ has zero average in $\mathcal{B}$. The Poincaré inequality implies that the sequence $\left\{u_{k}\right\}$ is bounded in $W^{1, p}(\mathcal{B})$. Then the compact embedding of $W^{1, p}$ into $L^{q}$, for $1 \leq q<p *=\frac{3 p}{3-p}$, yields

$$
u_{k} \rightarrow u \text { in } L^{q}(\mathcal{B}),
$$

and hence $F=D u$ in $\mathcal{B}$. Moreover, since by Theorem $3.3 \operatorname{adj} D u_{k}=\operatorname{Adj} D u_{k}$, we have

$$
\int_{\Omega}\left(\operatorname{adj} F_{k}\right){ }_{1}^{1} \varphi d x=-\int_{\Omega} u_{k}^{2}\left(u_{k, 3}^{3},-u_{k, 2}^{3}\right) \cdot(\varphi, 2, \varphi, 3) d x .
$$

On the other hand, since $p>3 / 2$, the strong convergence (3.3) holds for $q=3$ and therefore we can pass to the limit on the right hand side of (3.4)

$-\int_{\Omega} u_{k}^{2}\left(u_{k, 3}^{3},-u_{k, 2}^{3}\right) \cdot(\varphi, 2, \varphi, 3) d x \rightarrow-\int_{\Omega} u^{2}\left(u_{, 3}^{3},-u_{, 2}^{3}\right) \cdot(\varphi, 2, \varphi, 3) d x=\left\langle(\operatorname{Adj} D u)_{1}^{1}, \varphi\right\rangle$. 
Thus $A_{1}^{1}=(\operatorname{Adj} D u)_{1}^{1}$ (in the sense of distributions in $\left.\mathcal{B}\right)$. The above argument can be applied to each component of adj $F_{k}$. Thus $\operatorname{Adj} D u=A$ and in particular Adj $D u \in L^{1}(\mathcal{B})$. It follows from Theorem 3.3 that adj $D u=\operatorname{Adj} D u=A$.

Similarly

$$
\int_{\Omega} \operatorname{det} D u_{k} \varphi d x=-\int_{\Omega} u_{k}^{1}\left(\operatorname{adj} D u_{k}\right)^{1} \cdot D \varphi d x \rightarrow-\int_{\Omega} u^{1}(\operatorname{adj} D u)^{1} \cdot D \varphi d x
$$

and therefore, again by Theorem 3.3, $\operatorname{Det} D u=\operatorname{det} D u=D$ a.e. in $\mathcal{B}$, which implies $\operatorname{det} F=D$ a.e. in $\Omega$.

Finally observe that $F$ satisfies the constraint (i) since the latter is closed under weak convergence in $L^{p}$.

Remark 3.5 If $\Omega$ is a simply connected set, then (ii) is equivalent to the following condition:

for every smooth surface $\mathcal{S} \subset \Omega$ such that $\partial \mathcal{S}=\Gamma, \quad F=D u$ with $u \in \mathcal{E}(\Omega \backslash \mathcal{S})$.

In such a case, in the proof of Theorem 3.4, one can prove the convergence of (adj $F_{k}$, det $F_{k}$ ) directly in $\mathcal{D}^{\prime}(\Omega \backslash \mathcal{S})$, for any surface $\mathcal{S}$ with $\partial \mathcal{S}=\Gamma$.

Also, observe that if we required (3.5) to hold only for one given surface instead of every surface having $\Gamma$ as its boundary, then the set of admissible fields would be larger (see Example 3.6) and we might therefore get, at least in principle, a smaller minimum.

The following example shows that in general $u \in \mathcal{E}(\Omega \backslash \mathcal{S}) \cap W^{1, p}\left(\Omega, \mathbb{R}^{3}\right)$ does not necessarily imply $u \in \mathcal{E}(\Omega)$.

Example 3.6 Let $\phi: \mathbb{R}^{2} \rightarrow \mathbb{R}^{2}$ be given by

$$
\phi\left(x^{\prime}\right)=\frac{x^{\prime}}{\left|x^{\prime}\right|}, \quad x^{\prime}=\left(x_{1}, x_{2}\right) .
$$

It can be easily checked that $\phi \in W_{\text {loc }}^{1, p}\left(\mathbb{R}^{2}, \mathbb{R}^{2}\right)$ for any $p<2$ and that the distributional determinant Det $D \phi$ has an atom at $x^{\prime}=0$ :

$$
\text { Det } D \phi=\pi \delta_{0} \text {. }
$$

Then let $\eta \in C_{0}^{\infty}((-1,1)), \eta=1$ in $\left(-\frac{1}{2}, \frac{1}{2}\right)$, and define $u: \mathbb{R}^{3} \rightarrow \mathbb{R}^{3}$ in the following way

$$
u\left(x_{1}, x_{2}, x_{3}\right):=\left(\phi\left(x_{1}, x_{2}\right) \eta\left(x_{3}\right), 0\right) .
$$

The function $u$ satisfies for any $p<2$

$$
\begin{aligned}
& u \in W_{\text {loc }}^{1, p}\left(\mathbb{R}^{3}, \mathbb{R}^{3}\right), \\
& \text { Det } D u=\operatorname{det} D u=0 \text { a.e. in } \mathbb{R}^{3}, \\
& \text { Adj } D u=\operatorname{adj} D u \in L_{\text {loc }}^{p}\left(\mathbb{R}^{3} \backslash l\right), \text { where } l=\left\{(0,0, t) \in \mathbb{R}^{3}: t \in(-1,1)\right\}, \\
& (\text { Adj } D u)_{33}=\pi \eta^{2}\left(x_{3}\right) \mathcal{H}^{1}\left\llcorner\left\{x^{\prime}=0\right\} \text { in } \mathcal{D}^{\prime}\left(\mathbb{R}^{3}\right) .\right.
\end{aligned}
$$

Therefore for any ball $\mathcal{B}$ centered at the origin we have that $u \in \mathcal{E}(\mathcal{B} \backslash l)$, but $u \notin \mathcal{E}(\mathcal{B})$.

\section{Minimization over Cartesian maps}

In the present section we propose an alternative approach to the problem of minimizing (1.7) based on the theory of Cartesian maps. We first recall some basic definitions and results. 
Let $V \subset \mathbb{R}^{3}$ be an open set. We introduce the space $\mathcal{D}^{3}\left(V \times \mathbb{R}^{3}\right)$ of smooth three-forms with compact support in $V \times \mathbb{R}^{3}$ and the dual space $\mathcal{D}_{3}\left(V \times \mathbb{R}^{3}\right)$ of three-dimensional currents in $V \times \mathbb{R}^{3}$ (see [4,7], vol. I, for a comprehensive treatment on this subject). We say that the sequence $\left\{T_{k}\right\} \subset \mathcal{D}_{3}\left(V \times \mathbb{R}^{3}\right)$ converges weakly to $T \in \mathcal{D}_{3}\left(V \times \mathbb{R}^{3}\right), T_{k} \rightarrow T$, if

$$
T_{k}(\omega) \rightarrow T(\omega) \quad \forall \omega \in \mathcal{D}^{3}\left(V \times \mathbb{R}^{3}\right) .
$$

For $p \geq 1$ let

$$
\mathcal{A}^{p}\left(V, \mathbb{R}^{3}\right):=\left\{u \in W^{1, p}\left(V, \mathbb{R}^{3}\right): \operatorname{det} D u,|\operatorname{adj} D u| \in L^{p}(V)\right\},
$$

and for $u \in \mathcal{A}^{p}\left(V, \mathbb{R}^{3}\right)$ we set

$$
\|u\|_{\mathcal{A}^{p}}:=\|u\|_{L^{p}(V)}+\||M(D u)|\|_{L^{p}(V)},
$$

where $M(F)=(F, \operatorname{adj} F, \operatorname{det} F)$. We remark that $\mathcal{A}^{p}\left(V, \mathbb{R}^{3}\right)$ is not a linear space and $\|\cdot\|_{\mathcal{A}}$ is not a norm. Also, observe that our definition of $\mathcal{A}^{p}$ slightly differs from that given in [6]. We say that a sequence $\left\{u_{k}\right\}$ in $\mathcal{A}^{p}\left(V, \mathbb{R}^{3}\right)$ converges weakly in $\mathcal{A}^{p}\left(V, \mathbb{R}^{3}\right)$ to $u \in \mathcal{A}^{p}\left(V, \mathbb{R}^{3}\right)$

$$
u_{k} \rightarrow u \text { in } \mathcal{A}^{p}\left(V, \mathbb{R}^{3}\right)
$$

if and only if

$$
\begin{aligned}
& u_{k} \rightarrow u \text { strongly in } L^{p}\left(V, \mathbb{R}^{3}\right), \\
& M_{I}^{J}\left(D u_{k}\right) \rightarrow M_{I}^{J}(D u) \text { weakly in } L^{p}(V),
\end{aligned}
$$

for all $I, J$ with $1 \leq|I|=|J| \leq 3$.

For $u \in \mathcal{A}^{p}\left(V, \mathbb{R}^{3}\right)$ one can define the three-current $G_{u}$ carried by the graph of $u$ by integrating any form $\omega \in \mathcal{D}^{3}\left(V \times \mathbb{R}^{3}\right)$ over the graph of $u, \mathcal{G}_{u}$, i.e.,

$$
G_{u}(\omega):=\int_{\mathcal{G}_{u}} \omega=\sum_{|I|+|J|=3} \sigma(I, \bar{I}) \int_{V} h_{I J}(x, u(x)) M_{\bar{I}}^{J}(D u(x)) d x,
$$

for any three-form $\omega$

$$
\omega(x, y)=\sum_{|I|+|J|=3} h_{I J}(x, y) d x^{I} \wedge d y^{J}
$$

where $h_{I J} \in C_{0}^{\infty}\left(V \times \mathbb{R}^{3}\right)$ and $\sigma(I, \bar{I})$ is the sign of the permutation which reorders the multiindex $(I, \bar{I})$ in its natural order. The boundary of $T \in \mathcal{D}_{3}\left(V \times \mathbb{R}^{3}\right)$ is defined as the two-current

$$
\partial T(\eta):=T(d \eta), \quad \forall \eta \in \mathcal{D}^{2}\left(V \times \mathbb{R}^{3}\right) .
$$

If $u$ is a smooth map, then the Stokes theorem implies that $G_{u}$ has no boundary in $V \times \mathbb{R}^{3}$ :

$$
\partial G_{u}(\eta)=0 \quad \forall \eta \in \mathcal{D}^{2}\left(V \times \mathbb{R}^{3}\right),
$$

a condition which is clearly preserved by weak convergence (4.1). We can now introduce the class of $p$-Cartesian maps

$$
\operatorname{cart}^{p}\left(V, \mathbb{R}^{3}\right):=\left\{u \in \mathcal{A}^{p}\left(V, \mathbb{R}^{3}\right): \partial G_{u}\left\llcorner V \times \mathbb{R}^{3}=0\right\} .\right.
$$

The condition that the current associated with the graph of a Cartesian map is boundaryless ensures that the set $\operatorname{cart}^{p}\left(V, \mathbb{R}^{3}\right)$ is closed under weak convergence in $L^{p}$ of $u$ and of all minors of $D u$, as clarified by the following theorem. 
Theorem 4.1 If $u_{k}$ and $u$ belong to $\operatorname{cart}^{p}\left(V, \mathbb{R}^{3}\right), p \geq 1$, and

$$
\begin{aligned}
& u_{k} \rightarrow u \text { weakly in } L^{p}\left(V, \mathbb{R}^{3}\right), \\
& M_{I}^{J}\left(D u_{k}\right) \rightarrow v_{I}^{J} \text { weakly in } L^{p}(V),
\end{aligned}
$$

for all $I, J$ with $1 \leq|I|=|J| \leq 3$, then

$$
v_{I}^{J}(x)=M_{I}^{J}(D u(x)) \text { a.e. } x \in \Omega .
$$

Remark 4.2 The proof of the above theorem can be found in [6, vol. I, Sect. 3.3.3, Theorem 1]. Here we only remark that (4.3) is a consequence of the fact that the assumptions (4.2) imply the convergence of graphs $G_{u_{k}} \rightarrow G_{u}$ in $\mathcal{D}_{3}\left(V \times \mathbb{R}^{3}\right)$ which in turn relies on the Federer-Fleming closure theorem [5].

The sequential weak compactness of bounded sets in $L^{p}, p>1$, together with Theorem 4.1 yields the following compactness result.

Theorem 4.3 Let $\left\{u_{k}\right\}$ be a sequence of maps in $\operatorname{cart}^{p}\left(V, \mathbb{R}^{3}\right), p>1$. Suppose that

$$
\sup _{k}\left\|u_{k}\right\|_{\mathcal{A}^{p}}<\infty .
$$

Then there exists a subsequence $\left\{u_{k_{i}}\right\}$ of $\left\{u_{k}\right\}$ and a map $u \in \operatorname{cart}^{p}\left(V, \mathbb{R}^{3}\right)$ such that

$$
u_{k_{i}} \rightarrow u \text { in } \mathcal{A}^{p}\left(V, \mathbb{R}^{3}\right) \text {. }
$$

For the proof of Theorem 4.3 we refer the reader to [6, vol. I, Sect.3.3.3, Theorem 2].

Remark 4.4 In contrast with the case $p=1$, for $p>1$ the weak convergence of a sequence $\left\{u_{k}\right\}$ in $\operatorname{cart}^{p}\left(V, \mathbb{R}^{3}\right)$ is equivalent to the convergence of the graphs $G_{u_{k}}$ in $\mathcal{D}_{3}\left(V \times \mathbb{R}^{3}\right)$ together with the equiboundedness of the norms $\left\|u_{k}\right\|_{\mathcal{A}^{p}}$. This is not true for $p=1$ and indeed Theorem 4.3 is false in this case. More precisely, when $p=1$, by (4.4) we can only infer the existence of a subsequence $\left\{u_{k_{i}}\right\}$, a function $u \in B V\left(V, \mathbb{R}^{3}\right)$ and a current $T \in \mathcal{D}_{3}\left(V \times \mathbb{R}^{3}\right)$ such that

$$
\begin{aligned}
& u_{k_{i}} \rightarrow u \text { weakly in } B V\left(V, \mathbb{R}^{3}\right), \\
& M_{I}^{J}\left(D u_{k}\right) \rightarrow \mu_{I}^{J} \text { as measures, } \\
& G_{u_{k}} \rightarrow T \text { in } \mathcal{D}_{3}\left(V \times \mathbb{R}^{3}\right),
\end{aligned}
$$

but in general $T \neq G_{u}$ and $u$ and $\mu_{I}^{J}$ are only partially related.

Theorem 4.3 allows us to reformulate the minimization problem for (1.7) on a different set of fields. More precisely, in the definition of $\mathcal{F},(3.1)$, we may replace the set $\mathcal{E}(\mathcal{B})$ in (ii) by $\operatorname{cart}^{p}\left(\mathcal{B}, \mathbb{R}^{3}\right)$. We denote by (ii') the new assumption. Consequently we define

$$
\widetilde{\mathcal{F}}:=\left\{F \in L^{p}\left(\Omega, \mathbb{M}^{3 \times 3}\right): \text { (i) and (ii')hold }\right\} .
$$

Remark 4.5 It is known that the condition required in (ii') is stronger than that in (ii). More precisely, by Example 1 in Sect. 3.2.3 of Vol. I of [7], it follows that

$$
\begin{aligned}
\operatorname{cart}^{p}\left(V, \mathbb{R}^{3}\right) \subsetneq & \left\{u \in W^{1, p}\left(V, \mathbb{R}^{3}\right): \operatorname{adj} D U=\operatorname{Adj} D u \in L^{p}\left(V, \mathbb{M}^{3 \times 3}\right),\right. \\
& \left.\operatorname{det} D u=\operatorname{Det} D u \in L^{p}(V)\right\} .
\end{aligned}
$$

Consequently we find that the set $\widetilde{\mathcal{F}}$ is a proper subset of $\mathcal{F}$ :

$$
\widetilde{\mathcal{F}} \subsetneq \mathcal{F} \text {. }
$$


We now state the existence theorem on the set $\widetilde{\mathcal{F}}$ which, unlike Theorem 3.4, holds for any $1<p<2$.

Theorem 4.6 Let $1<p<2$. Assume that there exists $F \in \widetilde{\mathcal{F}}$ such that $\mathcal{W}(F)$ is finite. Then the functional $\mathcal{W}$ attains its minimum on $\widetilde{\mathcal{F}}$.

Proof The proof is an immediate consequence of Theorem 4.3.

Remark 4.7 Observe that a remark similar to Remark 3.5 also holds in the setting of Cartesian maps. Indeed the function $u$ constructed in Example 3.6 is smooth far away from $l$ and it therefore belongs to $\operatorname{cart}^{p}\left(V \backslash l, \mathbb{R}^{3}\right)$ for every open bounded set $V \subset \mathbb{R}^{3}$. However, if $V \cap l \neq \emptyset$, then $u \notin \operatorname{cart}^{p}\left(V, \mathbb{R}^{3}\right)$ as $\partial G_{u}\left\llcorner V \times \mathbb{R}^{3} \neq 0\right.$.

\section{References}

1. Ambrosio, L., Fusco, N., Pallara, D.: Functions of bounded variation and free discontinuity problems. Oxford, UP (2000)

2. Ball, J.M.: Convexity conditions and existence theorems in nonlinear elasticity. Arch. Ration. Mech. Anal. 63, 337-403 (1976)

3. Conti, S., De Lellis, C.: Some remarks on the theory of elasticity for compressible Neohhookean materials. Ann. Sci. Norm. Super. Pisa Cl. Sci. 3, 521-549 (2003)

4. Federer, H.: Geometric Measure Theory. Springer, New York (1969)

5. Federer, H., Fleming, W.: Normal and integral currents. Ann. Math. 72, 458-520 (1960)

6. Giaquinta, M., Modica, G., Souček, J.: Cartesian currents, weak diffeomorphisms and existence theorems in nonlinear elasticity. Arch. Ration. Mech. Anal. 106, 97-159 (1989). Erratum and addendum. Arch. Ration. Mech. Anal. 109, 385-392 (1990)

7. Giaquinta, M., Modica, G., Souček, J.: Cartesian Currents in the Calculus of Variations I and II. Springer, Berlin (1998)

8. Hirth, J.P., Lothe, J.: Theory of Dislocations. McGraw-Hill, New York (1968)

9. Müller, S.: Det=det. A Remark on the Distributional Determinant. C. R. Acad. Sci. Paris 311, 13-17 (1990)

10. Ortiz, M.: Lectures at the Vienna summer school on microstructures. Vienna, 25-29 September, (2000)

11. Phillips, R.: Crystals, Defects and Microstructures: Modeling Across Scales. Cambridge University Press, New York (2001)

12. Šverák, V.: Regularity properties of deformations with finite energy. Arch. Ration. Mech. Anal. 100, 105127 (1988) 